Laudatio

\section{Gotthard von Klinggräff und Jürgen Gebhardt - 160 Lebens- und 94 Schalljahre!}

Nun hat auch Gotthard von Klinggräff kurz nach seinem Freund und Alter Ego Jürgen Gebhardt den Schallkopf sorgfältig abgewischt und endgültig ins vorgesehene Fach zurückgelegt. Dem Neupräsidenten der DEGUM, Prof. J. Menzel, und dem Sektionsleiter der Inneren Medizin, Dr. Th. Müller, ist es ein Anliegen, die beiden Väter des Hamburger „Internistischen“ Ultraschalls würdigen zu lassen. Wir haben diese Aufgabe als langjährige Mitstreiter und Freunde gerne übernommen.

Als Jungspund in der Rettenmaier'schen Ultraschallschule bin ich den beiden 1974 beim 1. Böblinger Ultraschallkurs begegnet. Gotthard fiel unter den etwa 15 Kursteilnehmern bereits damals nicht nur durch seine attraktive Erscheinung, sondern auch durch seine Kommunikationsfähigkeit auf. Jürgen strahlte vor allem Ruhe, Ernsthaftigkeit und hanseatisches Understatement aus. Dieses Duo muss auch G. Rettenmaier gefallen haben. Entscheidend war ihr enormes und seriöses Interesse an der neuen Methode - die Sonografie wurde damals oft als eine Art Kaffeesatzlesen verspottet - und Rettenmaier konnte begeisterungsfähige Jünger wahrlich gebrauchen. Bald sprach er von seinen „Hamburger Metastasen“.
Auf Anregung ihres verehrten klinischen Lehrers Prof. Hornbostel sind Gotthard und Jürgen sehr früh in den praktischen Ultraschall eingestiegen, und sie haben es tatsächlich geschafft, die damalige Nischenmethode in den 1970er-Jahren trotz eingeschränkter Bildqualität und außerhalb universitärer Strukturen in Hamburg einzuführen und durchzusetzen. Als Mitbegründer des ASHI (Arbeitskreis Sonografie Hamburger Internisten) organisierten sie ab 1979 Sonografiekurse und hochkarätige US-Fortbildung in Hamburg. Gemeinsam prägten sie Flair und Stil der Kurse.

Die regelmäßig 1/2-jährlich stattfindenden Kolloquien führten das „Who is Who“ der sonografischen Kunst nach Hamburg. Die Referenten wussten die Einladungen als Auszeichnung zu schätzen - und kamen gern, obwohl es anschließend „nur“ Kartoffelsalat mit Würstchen gab. Ausbildung und Fortbildung auf hohem Niveau hatten Vorrang.

Gotthard v. Klinggräff und Jürgen Gebhardt waren vermutlich die ersten, die die Botschaft vom schnellen B-Bild erfolgreich nach Venezuela und Brasilien exportierten.

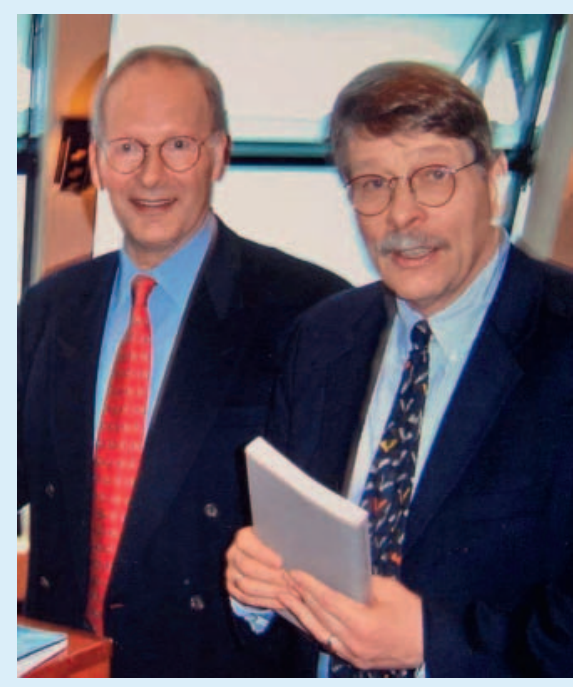

Jürgen Gebhardt (li.) und Gotthard von Klinggräff (re.). Foto: privat.

Nach regelmäßigen Treffen bei Fortbildungsveranstaltungen und mehreren Dreiländertreffen wurden wir Freunde. Wenn man Gotthard bei einer Ultraschallveranstaltung traf, war Jürgen meist nicht weit entfernt. So unterschiedlich die Freunde waren, so perfekt ergänzten sie sich. Gotthard war eher der begeisterungsfähige Initiator und Jürgen sorgte immer mit viel Einsatz für eine fehlerfreie Umsetzung. 
Ein Höhepunkt war unsere Zeit als Kongresspräsidenten des Dreiländertreffens 1989 in Hamburg. Gemeinsam schafften wir es, in verlässlicher Arbeitsteilung zusammen mit Jochen Hackelöer einen großen, unvergesslichen Kongress zu organisieren. Es kamen mehr als 2000 Teilnehmer, und erstmals boten wir ohne Terminüberschneidung zum wissenschaftlichen Programm kostenfreie Fortbildungsvorträge an. Den Höhepunkt bildete der Festabend mit mehr als $1200 \mathrm{Be}$ suchern in der Fischauktionshalle, mit 2 Bühnen und Live Bands aus Liverpool. Es wurde ein spektakulärer Festabend, ganz nach dem Geschmack der jungen wilden Schaller. Unser ,junges Präsidententeam“ schien der Zeit voraus, wir diskutierten lebhaft, bündelten unsere Stärken, keiner profilierte sich auf Kosten der anderen. Und alles lief wieder mal fehlerfrei, dank Jürgen, unserem ruhenden Pol.

Gotthard von Klinggräff hat zudem in seiner Berufskarriere viel für die DEGUM geleistet. 5 Jahre Vorstandsarbeit als Sekretär brachten ihm viel Anerkennung. Für seine Zuverlässigkeit und geschickte Art Interessen auszugleichen wurde er zurecht mit der Verdienstmedaille der DEGUM ausgezeichnet.

Gotthard war ein geschätzter Redner auf vielen DEGUM-Kongressen und für mich zudem ein wichtiger Koautor, der pünktlich seine bestens ausgearbeiteten Manuskripte mit hervorragendem Bildmaterial lieferte. Die 1980 gegründete Zeitschrift „Ultraschall in der Medizin“ unterstützte er von Anfang an als Autor und auch später, als die Zeitschrift als Organ der DEGUM „wackelte“. Auch dafür gilt ihm mein besonderer Dank.

Unsere Freundschaft erstreckte sich nicht nur auf den Ultraschall, sondern wurde auch in den Jahren nach unserer Pensionierung weiter gepflegt, ein aktuelles Treffen auf Föhr hat leider Corona vereitelt.

Für die beiden muss angesichts ihrer Meriten und ihres Engagements - in Analogie zu den Lichtjahren - der Begriff der Schalljahre eingeführt werden. So gemessen, mit je 47 Schalljahren, haben sie mich deutlich in den Schallschatten gestellt - das wird ihnen so leicht keiner nachmachen!

\section{Karlheinz Seitz, Sigmaringen}

Meine erste Begegnung mit Gotthard von Klinggräff liegt etwa 15 Jahre zurück. Ich war noch relativ neu in Hamburg, Gotthards Namen als langjähriger exzellenter Ultraschaller und einer der Protagonisten der „Hamburger Sono-Schule“ war aber auch an meiner bisherigen Wirkungsstätte in Südostbayern bestens bekannt.

Wir trafen uns an einem dunklen Winternachmittag in seinem nur spärlich beleuchteten Zimmer - und ich erhielt als erstes eine nicht allzu kurze, aber sehr unterhaltsame Unterweisung über die ausgesprochen verschlungenen Wege, wie im damals noch Landesbetriebskrankenhaus der Stadt Hamburg eine neue Glühbirne bestellt und geliefert werden konnte. Gotthard kann wunderbar erzählen, mit ein paar kleinen, manchmal leicht spöttischen Sidesteps hier, ein paar eingestreuten Anekdötchen dort - eine Fähigkeit, die ihm sowohl als Vortragender wie als Kursveranstalter und Moderator zahlreicher Ultraschallveranstaltungen ein interessiert zuhörendes, dankbares und treues Publikum beschert hat.

Wenig später lernte ich Jürgen Gebhardt kennen, zu jener Zeit als Leitender Oberarzt kommissarischer Leiter des renommierten AK Barmbek und zusammen mit Gotthard nicht nur Gründervater, sondern seit Jahren engagierter, kenntnisreicher und zuverlässig treibender Motor des oben schon erwähnten ASHI. Und wenn Gotthard passend zu seinen immer noch gepflegten automobilen Vorlieben immer wieder gerne den Turbo einlegen wollte, sorgte Jürgen mit seinem besonnenen und vorausschauenden Wesen dafür, dass das nur dann geschah, wenn die Straßen- und Sichtverhältnisse entsprechend waren - ein perfektes Duo eben. Ich durfte erfahren, dass die von den beiden langjährig geprägte Hamburger Ultraschall-„Szene“ eine ebenso hochkompetente wie engagierte, offene und Allüren-freie Gruppe ohne irgendwelche Abgrenzungstendenzen war und ist. Der von den beiden vorgelebte „Hamburger Sono-Spirit“ war immer: Ultraschall mit und unter Freunden, mit selbstverständlicher gegenseitiger Unterstützung, wo auch immer sie benötigt wurde.
Die erste Tagung des Arbeitskreises datiert vom Juni 1979. In den Programmarchiven des ASHI findet sich Jürgen als Organisator der Tagung mit einer „Video(!)aufzeichnung: Mitralstenose, Perikarderguss“, Gotthard mit einem Beitrag „Retroperitoneale Metastasierung“ - vielleicht schon damals ein Anklang auf den Hang zur technischen Perfektion bei dem einen, auf das spätere langjährige „Hobby“, die differenzierte Sonografie des Hodens, bei dem anderen.

Die gut besuchten ASHI-Abende, im weiteren Verlauf jeweils 1-mal im Jahr von Jürgen in Barmbek und Gotthard in Harburg organisiert, waren über Jahrzehnte gemeinsamer Treffund Fixpunkt der Hamburger Sono-Fangemeinde. Die Programme der ersten Jahre weisen Beiträge aus verschiedenen Hamburger Kliniken und Praxen mit Themen wie „Eindrücke von einem computergesteuerten B-Scanner“ oder „Drainage eines Leberabszesses unter Ultraschallkontrolle“ aus und belegen die enormen Entwicklungen, die die beiden miterlebt, mitgestaltet und mitmoderiert haben. 40 Jahre nach der Auftaktveranstaltung organisierte Gotthard sein letztes ASHI-Kolloquium im Dezember 2019 - es war das 74.

Aufgrund ihrer großen Verdienste um den Ultraschall hat der ASHI Jürgen Gebhardt und Gotthard von Klinggräff zu Ehrenvorsitzenden ernannt. Beide werden in diesem Jahr im Abstand von nur 1 Woche 80 Jahre alt, und beide haben bis vor kurzem regelmäßig den Schallkopf geführt und ebenso ästhetische wie präzise Bilder und Befunde erstellt. Sie haben ihre Begeisterung für den Ultraschall an die nächsten Generationen (der Plural sei angesichts des Lebensalters erlaubt) engagiert, hochkompetent und mit unverwüstlichem Mecklenburger Charme, respektive Artländer Seriosität und Bodenständigkeit weitergegeben. Die Hamburger UltraschallCommunity kann sich ihre langjährigen Inspiratoren zwar ohne Ultraschall eigentlich genauso wenig vorstellen, wie umgekehrt die Hamburger Sono-Gemeinde ohne die beiden - bedankt sich aber von Herzen für die langjährige Prägung, Führung und Freundschaft!

\section{Guntram Lock, Hamburg}

Zu unserem großen Bedauern müssen wir mitteilen, dass Jürgen Gebhardt nach Drucklegung dieses Textes verstorben ist. Unser Mitgefühl gilt seiner Familie. 